\title{
Will cognitive mapping hinder Alzheimer's Disease?
}

\author{
May Yuan ${ }^{\mathrm{a}, *}$, Kristen Kennedy ${ }^{\mathrm{b}}$ \\ ${ }^{a}$ Geospatial Information Sciences, University of Texas at Dallas,myuan@utdallas.edu \\ ${ }^{b}$ Behavioral and Brain Sciences, University of Texas at Dallas, kristen.kennedy1@utdallas.edu \\ * Corresponding author
}

Keywords: spatial cognition, cognitive mapping, environmental complexity, Alzheimer's Disease, machine learning

\section{Abstract:}

Golledge (1992) distinguished three categories of spatial knowledge: landmark, route, and survey. Place recognition and wayfinding constitute the essential tasks to acquire, accumulate, and communicate spatial knowledge about a neighborhood, a city, or broader geography. We recognize landmarks or prominent features as reference locations in an environment and travel through the environment to learn routes that connect these places. While we can directly perceive figural and vista spaces, we cannot comprehend environmental space without locomotion and information experienced or learned over time, nor can we appreciate geographic space without symbolic representations or maps (Montello 1993). Survey knowledge is key in learning environmental and geographical spaces where allocentric frames of reference, independent of the observer's location and orientation, is a more efficient strategy to learn and communicate spatial knowledge than egocentric alternatives based on our body positions and direct sensory input.

Spatial representations in our neural system correspond to the egocentric and allocentric frames of reference. Neurons in the parietal cortex have receptive fields responsive to multiple sensory modalities and encode spatial information in egocentric frames of reference (Andersen, 1997; Colby, 1998). A system of spatial cells in the hippocampal formation has the neural firing fields independent of one's body but anchored to the external environment, hence an allocentric reference frame. The system includes place cells, grid cells, boundary cells, and head direction cells. These cells provide the basis for cognitive map building and long-term memory encoding for spatial relationships among places, routes connecting places, and resources, hazards, other properties, or experiences at these places (Hartley et al. 2014). The firing location in grid cells reflects the scale of an environment. Multiple firings in a triangular or hexagonal pattern aligned with visual features or display bounds suggest allocentric functions for estimating spatial relations and path integration (Giocomo et al. 2011). Nevertheless, allocentric tasks can involve egocentric components such that we need to have egocentric information to make a movement. The posterior cingulate cortex and retrosplenial cortex enable us to switch between egocentric and allocentric frames of reference, but MCI/AD patients show declines in the allocentric spatial frames and switching impairments, yet no specific switching deficit apparent in healthy elderly (Colombo et al. 2017). Topographic disorientation is an important early sign of aMCI (i.e., MCI leading to AD) and AD. Allocentric deficits prevail in both aMCI and AD patients and disable them to encode an allocentric representation and use it with or translate it to an egocentric representation (Serino et al. 2014).

The neural system of spatial cells subserves allocentric frames of reference. People in a more complex environment rely more on allocentric frames for place recognition and wayfinding for more efficient encoding of spatial information in the forms of cognitive maps. The process exercises spatial cells in the hippocampal formation and may maintain or strengthen the firing functions of place cells, grid cells, boundary cells, and head orientation cells. Subsequently, cognitive map building may lead to health benefits that can delay AD onset or slow AD progression. To date, there is no pharmaceutical cure for AD. Evidence that shows AD benefits from cognitive map building can offer cost-effective non-pharmaceutical intervention for AD mitigation. This study uses 15-years of annual survey data from the US National Alzheimer's Coordination Center (NACC) on normal, MCI, and AD patients. We selected 21,902 NACC subjects who had multiple annual visits and stayed in the same 3-digit zipcode zones throughout these visits.

Our methodology was based on the following conceptual framework: (1) In the context of spatial cells for allocentric cognitive mapping, higher environmental complexity would correspond to more complex street networks and more POIs that could serve landmark functions; (2) Cognitive demand on allocentric frames of reference would simulate spatial cells and maintain or improve their firing fields and neural functions for allocentric cognition and navigation, leading to comparably less MCI or AD subjects in more complex 3-digit zipcode zones. The area coverage of the selected 3-digit zipcode zones varied significantly from slightly less than $0.01 \mathrm{~km}^{2}$ to more than $266,000 \mathrm{~km}$. To limit spatial heterogeneity, we selected only the lower $25 \%$ of 3-digit zipcode zones in size. The selection resulted in 89 3-digit zipcode zones with the largest area size of $1,059 \mathrm{~km}^{2}$ and a total of 17,264 NACC subjects. For each of the 89 zipcode zones, we calculated the percentages of NACC subjects characterized as normal, no dementia, to-dementia, dementia, no MCI, MCI, and AD. Then we standardized each of the percentages of cognitive measures across all the selected zipcode zones (Fig.1). The spatial distribution of the selected 89 zipcode zones and the cluster to which each zone belongs spreads across the continental US. We applied the agglomerative clustering method based on affinity propagation to identify clusters of zipcode zones based on their similarity among the eight standardized cognitive 
measures. The unsupervised clustering algorithm results in three zipcode clusters of varying cognitive measures: (0) low MCI/AD, (1) high MCI/AD, and (2) transition (Fig.2).

For each of the selected 3-digit zipcode zones, we extracted 27 street-network measures of edges and nodes for each census block and points of interest (POIs), serving as landmarks from SafeGraph POI database. We applied machine learning methods to predict the cognitive cluster (i.e., 0,1 , or 2 ) to which a given zipcode zone belongs based on only the network measures or only the POI measures. The complexity of network measured decreases from cluster0 (most complex), to cluster2, and to cluster1(simplest). Cluster1 was associated with long road segments, 3-way intersections, dead-ends, and extensive closures. Low network measures with cluster1 included a maximal pagerank index, average degree centrality, street density, and interaction four-way proportion. While the average numbers of POI per tract were comparable across the three clusters (64.29, 68.75 , and 63.32), higher percentages of tracts without any POIs appear in cluster1 $(15.36 \%)$ and cluster2 $(13.66 \%)$ than the percentage in cluster0 $(8.23 \%)$. In comparison, POIs in cluster0 had more durable good merchant wholesalers, motor vehicle dealers, warehouse clubs, cluster 1 has more waste treatment and disposal sites, and cluster2 has more gas stations and religious organizations. POIs in cluster1 were less likely used as landmarks for navigation than POIs in clusters 0 and 2.

We reserved $20 \%$ of data for testing and applied 5 -fold cross-validation with $80 \%$ of the data for training. Specifically, Support Vector Machine results in $\sim 60 \%$ prediction accuracy for all three cognitive clusters based on network measures and $\sim 50 \%$ prediction accuracy based on random-forest-based extreme gradient boost (XBG) for all three cognitive clusters based on POI measures (Fig.3). If these measures had no effect, the prediction accuracy would have approximated 33\%. Currently, we are seeking to identify the best predictors for environmental complexity and build a model that integrates networks, POIs, and covariates (e.g., demographics, gender, race, and education) to evaluate the overall effects of environmental complexity on $\mathrm{MCI}$ and $\mathrm{AD}$ prevalence.

Acknowledgements: The research is supported by (US) National Institute of Health (NIH) R21AG069267.

\section{References}

Andersen, R. A. (1997). Multimodal integration for the representation of space in the posterior parietal cortex. 8 .

Colby, C. L. (1998). Action-Oriented Spatial Reference Frames in Cortex. Neuron, 20(1), 15-24. 10.1016/S08966273(00)80429-8

Colombo, D., Serino, S., Tuena, C., Pedroli, E., Dakanalis, A., Cipresso, P., \& Riva, G. (2017). Egocentric and allocentric spatial reference frames in aging: A systematic review. Neuroscience \& Biobehavioral Reviews, 80, 605621. 10.1016/j.neubiorev.2017.07.012

Giocomo, L. M., Hussaini, S. A., Zheng, F., Kandel, E. R., Moser, M.-B., \& Moser, E. I. (2011). Grid Cells Use HCN1 Channels for Spatial Scaling. Cell, 147(5), 1159-1170. 10.1016/j.cell.2011.08.051

Golledge, R. G. (1992). Place recognition and wayfinding: Making sense of space. Geoforum, 23(2), 199-214. 10.1016/0016-7185(92)90017-X

Hartley, T., Lever, C., Burgess, N., \& O'Keefe, J. (2014). Space in the brain: How the hippocampal formation supports spatial cognition. Philosophical Transactions of the Royal Society B: Biological Sciences, 369(1635), 20120510. 10.1098/rstb.2012.0510

Montello, D. R. (1993). Scale and multiple psychologies of space. In A. U. Frank \& I. Campari (Eds.), Spatial Information Theory A Theoretical Basis for GIS (Vol. 716, pp. 312-321). Springer Berlin Heidelberg. 10.1007/3540-57207-4 21

Serino, S., Cipresso, P., Morganti, F., \& Riva, G. (2014). The role of egocentric and allocentric abilities in Alzheimer's disease: A systematic review. Ageing Research Reviews, 16, 32-44. 10.1016/j.arr.2014.04.004 